\title{
A special class of regular connections in Finsler geometry
}

\author{
A. Soleiman ${ }^{1,2}$
}

\section{Correspondence:}

amr.hassan@fsci.bu.edu.eg amrsoleiman@yahoo.com; asoliman@ju.edu.sa

'Department of Mathematics, Faculty of Sciences, Benha University, Benha, Egypt 2Department of Mathematics, Collage of Sciences and Arts in Gurayat, Jouf University, Sakakah, Kingdom of Saudi Arabia

\begin{abstract}
In this paper, we investigate intrinsically a special class of regular Finsler connections in the pullback formalism. Such connections are called $P^{1}$-connections. Important result concerning the nonlinear connection of these connections is obtained. Some examples of $P^{1}$-connections are given. One of these examples is investigated with some details.
\end{abstract}

Keywords: Finsler manifold, $P^{1}$-connection, Nonlinear connection, Barthel connection, Cartan connection, Berwald connection, Chern connection, Hashiguchi connection

MSC 2010: 53C60, 53B40, 58B20

\section{Introduction}

The theory of connections is an important field of research of differential geometry. It was initially developed to solve pure geometrical problems. In [1,2] and others, the theory of Finsler linear connections has been studied.

Intrinsic proofs of the existence and uniqueness theorems for the fundamental Finsler linear connections on the pullback bundle are investigated by [3-5].

The main purpose of this paper is to provide an intrinsic study for a special class of regular Finsler connections in the pullback bundle. These connections are called $P^{1}$ connections. It is shown that for these connections, the associated semi-spray coincides with the canonical spray and the associated nonlinear connection coincides with the Barthel connection. Some examples of $P^{1}$-connections are given. One of these example is investigated. The fundamental relations concerning the torsion and the curvature tensor fields associated with this example are obtained.

Finally, it should be pointed out that the present work is formulated in a coordinate-free form.

\section{Notation and preliminaries}

In this section, we give a brief account of the basic concepts of the pullback approach to intrinsic Finsler geometry necessary for this work. For more details, we refer to [2, 4-9]. We shall use the notations of [4].

In what follows, we denote by $\pi: \mathcal{T} M \longrightarrow M$ the subbundle of nonzero vectors tangent to $M, \mathfrak{F}(T M)$ the algebra of $C^{\infty}$ functions on $T M$, and $\mathfrak{X}(\pi(M))$ the $\mathfrak{F}(T M)$-module of differentiable sections of the pullback bundle $\pi^{-1}(T M)$. The elements of $\mathfrak{X}(\pi(M))$ will be called $\pi$-vector fields and will be denoted by barred letters $\bar{X}$. The tensor fields on

(c) The Author(s). 2019 Open Access This article is distributed under the terms of the Creative Commons Attribution 4.0 International License (http://creativecommons.org/licenses/by/4.0/), which permits unrestricted use, distribution, and reproduction in any medium, provided you give appropriate credit to the original author(s) and the source, provide a link to the Creative Commons license, and indicate if changes were made. 
$\pi^{-1}(T M)$ will be called $\pi$-tensor fields. The fundamental $\pi$-vector field is the $\pi$-vector field $\bar{\eta}$ defined by $\bar{\eta}(u)=(u, u)$ for all $u \in \mathcal{T} M$.

We have the following short exact sequence of vector bundles:

$$
0 \longrightarrow \pi^{-1}(T M) \stackrel{\gamma}{\longrightarrow} T(\mathcal{T} M) \stackrel{\rho}{\longrightarrow} \pi^{-1}(T M) \longrightarrow 0,
$$

with the well-known definitions of the bundle morphisms $\rho$ and $\gamma$. The vector space $V_{u}(\mathcal{T} M)=\left\{X \in T_{u}(\mathcal{T} M): d \pi(X)=0\right\}$ is the vertical space to $M$ at $u \in \mathcal{T} M$.

Let $D$ be a linear connection on the pullback bundle $\pi^{-1}(T M)$. We associate with $D$ the map $K: T \mathcal{T} M \longrightarrow \pi^{-1}(T M): X \longmapsto D_{X} \bar{\eta}$, called the connection map of $D$. The vector space $H_{u}(\mathcal{T} M)=\left\{X \in T_{u}(\mathcal{T} M): K(X)=0\right\}$ is called the horizontal space to $M$ at $u$. The connection $D$ is said to be regular if:

$$
T_{u}(\mathcal{T} M)=V_{u}(\mathcal{T} M) \oplus H_{u}(\mathcal{T} M) \forall u \in \mathcal{T} M .
$$

If $M$ is endowed with a regular connection, then the vector bundle maps $\gamma,\left.\rho\right|_{H(\mathcal{T} M)}$ and $\left.K\right|_{V(\mathcal{T} M)}$ are vector bundle isomorphisms. The map $\beta:=\left(\left.\rho\right|_{H(\mathcal{T} M)}\right)^{-1}$ will be called the horizontal map of the connection $D$.

The horizontal ((h)h-) and mixed ((h)hv-) torsion tensors of $D$, denoted by $Q$ and $T$ respectively, are defined by:

$$
Q(\bar{X}, \bar{Y})=\mathbf{T}(\beta \bar{X}, \beta \bar{Y}), \quad T(\bar{X}, \bar{Y})=\mathbf{T}(\gamma \bar{X}, \beta \bar{Y}) \quad \forall \bar{X}, \bar{Y} \in \mathfrak{X}(\pi(M)),
$$

where $\mathbf{T}$ is the (classical) torsion tensor field associated with $D$.

The horizontal (h-), mixed (hv-), and vertical (v-) curvature tensors of $D$, denoted by $R$, $P$, and $S$ respectively, are defined by:

$$
R(\bar{X}, \bar{Y}) \bar{Z}=\mathbf{K}(\beta \bar{X}, \beta \bar{Y}) \bar{Z}, \quad P(\bar{X}, \bar{Y}) \bar{Z}=\mathbf{K}(\beta \bar{X}, \gamma \bar{Y}) \bar{Z}, \quad S(\bar{X}, \bar{Y}) \bar{Z}=\mathbf{K}(\gamma \bar{X}, \gamma \bar{Y}) \bar{Z},
$$

where $\mathbf{K}$ is the (classical) curvature tensor field associated with $D$.

The contracted curvature tensors of $D$, denoted by $\widehat{R}, \widehat{P}$, and $\widehat{S}$ (known also as the (v)h-, (v)hv-, and (v)v-torsion tensors respectively), are defined by:

$$
\widehat{R}(\bar{X}, \bar{Y})=R(\bar{X}, \bar{Y}) \bar{\eta}, \quad \widehat{P}(\bar{X}, \bar{Y})=P(\bar{X}, \bar{Y}) \bar{\eta}, \quad \widehat{S}(\bar{X}, \bar{Y})=S(\bar{X}, \bar{Y}) \bar{\eta} .
$$

We terminate this section by some concepts and results concerning the Klein-Grifone approach to intrinsic Finsler geometry. For more details, we refer to [10-13].

A semispray is a vector field $X$ on $T M, C^{\infty}$ on $\mathcal{T} M$, and $C^{1}$ on $T M$, such that $\rho \circ X=\bar{\eta}$. A semispray $X$ which is homogeneous of degree 2 in the directional argument $([\mathcal{C}, X]=X)$ is called a spray.

Proposition 1 [13] Let $(M, L)$ be a Finsler manifold. The vector field $G$ on TM defined by $i_{G} \Omega=-d E$ is a spray, where $E:=\frac{1}{2} L^{2}$ is the energy function and $\Omega:=d d_{J} E$. Such a spray is called the canonical spray.

A nonlinear connection on $M$ is a vector 1 -form $\Gamma$ on $T M, C^{\infty}$ on $\mathcal{T} M$ and $C^{0}$ on $T M$, such that:

$$
J \Gamma=J, \quad \Gamma J=-J .
$$

The horizontal and vertical projectors $h_{\Gamma}$ and $v_{\Gamma}$ associated with $\Gamma$ are defined by $h_{\Gamma}:=$ $\frac{1}{2}(I+\Gamma)$ and $v_{\Gamma}:=\frac{1}{2}(I-\Gamma)$. 
Theorem 1 [12] On a Finsler manifold $(M, L)$, there exists a unique conservative homogenous nonlinear connection with zero torsion. It is given by:

$$
\Gamma=[J, G] \text {, }
$$

where $G$ is the canonical spray.

Such a nonlinear connection is called the canonical connection, the Barthel connection, or the Cartan nonlinear connection associated with $(M, L)$.

\section{$\boldsymbol{P}^{\mathbf{1}}$-connections}

In this section, we investigate intrinsically a special class of regular connections in Finsler geometry within the pullback formalism. These connections are called $P^{1}$-connections. It is shown that for these connections, the associated semi-spray coincides with the canonical spray and the associated nonlinear connection coincides with the Barthel connection. Some examples of $P^{1}$-connections are given. All of this section, we use the notions and results of [3].

Definition 1 Let $D$ be a regular connection on $\pi^{-1}(T M)$ with horizontal map $\beta$. Then, the semispray $S=\beta \bar{\eta}$ will be called the semispray associated with D. Moreover, the nonlinear connection $\Gamma=2 \beta \circ \rho-I$ will be called the nonlinear connection associated with $D$ and will be denoted by $\Gamma_{D}$.

Lemma 1 Let $D$ be a regular connection on $\pi^{-1}(T M)$ whose connection map is $K$ and whose horizontal map is $\beta$. If the (h)hv-torsion $T$ of $D$ has the property that $T(\bar{X}, \bar{\eta})=0$, then $K=\gamma^{-1}$ on $V(T M)$ and $\Gamma:=\beta \circ \rho-\gamma \circ K$ is a nonlinear connection on $M$ and coincides with the nonlinear connection associated with $D: \Gamma=\Gamma_{D}=2 \beta \circ \rho-I$, and in this case $h_{\Gamma}=h_{D}=\beta \circ \rho$ and $v_{\Gamma}=v_{D}=\gamma \circ K$.

Definition 2 [14] Let $(M, L)$ be a Finsler manifold and $g$ the Finsler metric defined by $L$.

(a) A vector (2) $\pi$-form $\omega$ is indicatory if and only if $\omega(\bar{X}, \bar{\eta})=0=\omega(\bar{\eta}, \bar{X})$ and $g(\omega(\bar{X}, \bar{Y}), \bar{\eta})=0$.

(b) A scaler (2) $\pi$-form $\omega$ is indicatory if and only if $\omega(\bar{X}, \bar{\eta})=0=\omega(\bar{\eta}, \bar{X})$.

We will study the Finsler connections defined by the set of axiom given in the next definition.

Definition 3 Let $(M, L)$ be a Finsler manifold. A regular connection $\bar{D}$ on $\pi^{-1}(T M)$ is called a $P^{1}$-connection if it satisfies the following conditions:

(a) $\bar{D}_{\bar{h} X} L=0$, for all $X \in \mathfrak{X}(\mathcal{T} M)$,

(b) the (h)hv-torsion $\bar{T}$ of $\bar{D}$ is symmetric and indicatory.

(c) the (h)h-torsion tensor $\bar{Q}$ of $\bar{D}$ vanishes,

(d) the (v)hv-torsion tensor $\widehat{\bar{P}}$ of $\bar{D}$ is symmetric and indicatory.

Examples Cartan and Berwald connections are $P^{1}$-connection [3]. Also Chern and Hashiguchi connections are $P^{1}$-connection [4].

Proposition 2 Let $\bar{D}$ be $P^{1}$-connection on $\pi^{-1}(T M)$ whose connection map is $\bar{K}$ and whose horizontal map is $\bar{\beta}$. Then, for all $\bar{X}, \bar{Y} \in \mathfrak{X}(\pi(M))$, we have: 
(a) $[\gamma \bar{X}, \gamma \bar{Y}]=\gamma\left(\bar{D}_{\gamma \bar{X}} \bar{Y}-\bar{D}_{\gamma \bar{Y}} \bar{X}\right)$

(b) $[\gamma \bar{X}, \bar{\beta} \bar{Y}]=-\gamma\left(\widehat{\bar{P}}(\bar{Y}, \bar{X})+\bar{D}_{\bar{\beta} \bar{Y}} \bar{X}\right)+\bar{\beta}\left(\bar{D}_{\gamma \bar{X}} \bar{Y}-\bar{T}(\bar{X}, \bar{Y})\right)$

(c) $[\bar{\beta} \bar{X}, \bar{\beta} \bar{Y}]=\gamma(\widehat{\bar{R}}(\bar{X}, \bar{Y}))+\bar{\beta}\left(\bar{D}_{\bar{\beta} \bar{X}} \bar{Y}-\bar{D}_{\bar{\beta} \bar{Y}} \bar{X}\right)$

Proof First we prove that the (v)v-torsion tensor $\widehat{\bar{S}}$ of a $P^{1}$-connection vanishes. Since a $P^{1}$-connection is regular and whose (h)hv-torsion tensor $\bar{T}$ has the property that $\bar{T}(\bar{X}, \bar{\eta})=0$ (Definition 3(b)), then, by Proposition 2 of [5], the $v$-curvature tensor $\bar{S}$ is given by:

$$
\begin{aligned}
\bar{S}(\bar{X}, \bar{Y}) \bar{Z}= & \left(\bar{D}_{\gamma \bar{Y}} \bar{T}\right)(\bar{X}, \bar{Z})-\left(\bar{D}_{\gamma \bar{X}} \bar{T}\right)(\bar{Y}, \bar{Z}) \\
& +\bar{T}(\bar{X}, \bar{T}(\bar{Y}, \bar{Z}))-\bar{T}(\bar{Y}, \bar{T}(\bar{X}, \bar{Z}))+\bar{T}(\widehat{\bar{S}}(\bar{X}, \bar{Y}), \bar{Z}) .
\end{aligned}
$$

Setting $\bar{Z}=\bar{\eta}$ into the above relation, noting that $\bar{T}$ is symmetric indicatory, gives

$$
\widehat{\bar{S}}=0 \text {. }
$$

Now, from (1) and Lemma 2.1 of [5], the result follows.

Theorem 2 Let $(M, L)$ be a Finsler manifold and $\bar{D}$ a $P^{1}$-connection. Then, the associated nonlinear connection $\bar{\Gamma}$ has the form:

$$
\bar{\Gamma}:=\bar{\beta} o \rho-\gamma o \bar{K} .
$$

Moreover, $\bar{\Gamma}$ coincides with the Barthel connection of $(M, L): \bar{\Gamma}=[J, G]$.

Proof Since the (h)hv-torsion of $\bar{D}$ satisfies $\bar{T}(\bar{X}, \bar{\eta})=0$, then, by Lemma 1, it follows that $\bar{\Gamma}:=\bar{\beta} o \rho-\gamma o \bar{K}$. Moreover, its projectors are $\bar{h}=\bar{\beta} o \rho$ and $\bar{v}=\gamma o \bar{K}$.

We prove that $\bar{\Gamma}$ enjoys the following properties:

$\bar{\Gamma}$ is conservative:

In fact, if $E=\frac{1}{2} L^{2}$, then

$$
d_{\bar{h}} E(X)=i_{\bar{h}} d E(X)=L(\bar{h} X \cdot L)=L \bar{D}_{\bar{h} X} L=0 . \quad(\text { by axiom }(a))
$$

Hence, the result follows.

$\bar{\Gamma}$ is homogenous of degree one:

$$
\begin{aligned}
{[\mathcal{C}, \bar{v}](X) } & =[\mathcal{C}, \gamma \circ \bar{K}](X)=[\mathcal{C}, \gamma \circ \bar{K}(X)]-\gamma \circ \bar{K}([\mathcal{C}, X]) \\
& =[\mathcal{C}, \gamma(\bar{K} X)]-\gamma(\bar{K}[\mathcal{C}, \bar{h} X+\bar{v} X])=[\mathcal{C}, \gamma(\bar{K} X)]-\gamma(\bar{K}[\mathcal{C}, \bar{v} X])-\gamma(\bar{K}[\mathcal{C}, \bar{h} X])
\end{aligned}
$$

From which, taking into account the fact that $[\mathcal{C}, \bar{v} X]$ is vertical and $\gamma o \bar{K}=i d_{V(T M)}$ and using Proposition 2, we get:

$$
\begin{aligned}
{[\mathcal{C}, \bar{v}](X) } & =-\gamma(\bar{K}[\mathcal{C}, \bar{h} X])=-\gamma o \bar{K}([\gamma \bar{\eta}, \bar{\beta} \rho X]) \\
& =-\gamma o \bar{K}\left\{-\gamma\left(\widehat{\bar{P}}(\rho X, \bar{\eta})+\bar{D}_{\bar{\beta} \rho X} \bar{\eta}\right)+\bar{\beta}\left(\bar{D}_{\gamma \bar{\eta}} \rho X-\bar{T}(\bar{\eta}, \rho X)\right)\right\} \\
& \left.=\gamma\left\{\left(\widehat{\bar{P}}(\rho X, \bar{\eta})+\bar{D}_{\bar{\beta} \rho X} \bar{\eta}\right)\right\} \bar{\beta}=0\right)=0 . \quad(\text { as } \widehat{\bar{P}} \text { is indicatory and } \bar{K} o \bar{\beta}=0)
\end{aligned}
$$

Therefore, $[\mathcal{C}, \bar{\Gamma}]=-2[\mathcal{C}, \bar{v}]=0$, which means that $\bar{\Gamma}$ is homogenous. 
As $J \bar{v}=0$ and $\bar{v} J=J$, we have

$$
\begin{aligned}
{[J, \bar{v}](X, Y)=} & {[J X, \bar{v} Y]+[\bar{v} X, J Y]+\bar{v} J[X, Y]+J \bar{v}[X, Y] } \\
& -J[\bar{v} X, Y]-J[X, \bar{v} Y]-\bar{v}[J X, Y]-\bar{v}[X, J Y] \\
= & J[\bar{h} X, \bar{h} Y]-\bar{v}[J X, \bar{h} Y]-\bar{v}[\bar{h} X, J Y] \\
= & J[\bar{\beta} \rho X, \bar{\beta} \rho Y]-\bar{v}[\gamma \rho X, \bar{\beta} \rho Y]+\bar{v}[\gamma \rho Y, \bar{\beta} \rho X] .
\end{aligned}
$$

Using Proposition 2, we get:

$$
\begin{aligned}
{[J, \bar{v}](X, Y)=} & J\left\{\gamma(\widehat{\bar{R}}(\rho X, \rho Y))+\bar{\beta}\left(\bar{D}_{\bar{h} X} \rho Y-\bar{D}_{\bar{h} Y} \rho X\right)\right\} \\
& -\gamma o \bar{K}\left\{-\gamma\left(\widehat{\bar{P}}(\rho Y, \rho X)+\bar{D}_{\bar{h} Y} \rho X\right)+\bar{\beta}\left(\bar{D}_{J X} \rho Y-\bar{T}(\rho X, \rho Y)\right)\right\} \\
& +\gamma o \bar{K}\left\{-\gamma\left(\widehat{\bar{P}}(\rho X, \rho Y)+\bar{D}_{\bar{h} X} \rho Y\right)+\bar{\beta}\left(\bar{D}_{J Y} \rho X-\bar{T}(\rho Y, \rho X)\right)\right\} .
\end{aligned}
$$

Since $\bar{K} o \bar{\beta}=0, \rho o \gamma=0, \bar{K} o \gamma=i d_{\mathfrak{X}(\pi(M))}, \rho o \bar{\beta}=i d_{\mathfrak{X}(\pi(M))}$ and $\widehat{\bar{P}}$ is symmetric, then the above relation implies that $[J, \bar{v}]=0$.

Hence $t:=\frac{1}{2}[J, \bar{\Gamma}]=-[J, \bar{v}]=0$.

From the above consideration, $\bar{\Gamma}=\bar{\beta} \circ \rho-\gamma \circ \bar{K}$ is a conservative torsion-free homogenous nonlinear connection. By the uniqueness of the Barthel connection (Theorem 1), it follows that $\bar{\Gamma}$ coincides with the Barthel connection $[, G]$.

In view of the above theorem and Definition 1, we obtain the following corollary:

Corollary 1 The semispray associated with a $P^{1}$-connection is a spray which coincides with the canonical spray.

We reconsider some results in references [3] and [4] with Theorem 2; we get the following corollary:

Corollary 2 Let $(M, L)$ be a Finsler manifold. The nonlinear connection associated with each of Cartan, Berwald, Chern, and Hashiguchi connections coincides with the Barthel connection.

Now, we will give an interesting example of $P^{1}$-connection called as $\widetilde{D}$-connection.

Theorem 3 Let $(M, L)$ be a Finsler manifold. There exists a unique regular connection $\widetilde{D}$ on $\pi^{-1}(T M)$ such that:

(a) $\widetilde{D}_{\widetilde{h} X} L=0$,

(b) $\widetilde{D}$ is torsion-free: $\widetilde{\mathbf{T}}=0$,

(c) The (v)hv-torsion tensor $\widehat{\widetilde{P}}$ of $\widetilde{D}$ has the form $\widehat{\widetilde{P}}(\bar{X}, \bar{Y})=-L T(\bar{X}, \bar{Y})$.

Such a connection is called the $\widetilde{D}$-connection associated with the Finsler manifold $(M, L)$.

Proof It is easy to show first that any regular connection $\widetilde{D}$ satisfying the conditions (a), (b), and (c) is necessarily a $P^{1}$-connection. Consequently, in view of Theorem 2, the nonlinear connection $\Gamma_{\widetilde{D}}$ associated with $\widetilde{D}$ coincides with the Barthel connection: $\Gamma_{\widetilde{D}}=[J, G]$. Moreover, $\widetilde{\beta}=\beta, \widetilde{K}=K, \widetilde{h}=h$, and $\widetilde{v}=v$, where $\beta, K, h$, and $v$ are 
the horizontal map, connection map, horizontal projector, and the vertical projector of Cartan connection $\nabla$, respectively.

Now, we prove the uniqueness. As $\widetilde{D}$ is a nonmetric linear connection on $\pi^{-1}(T M)$ with zero torsion, one can show that:

$2 g\left(\widetilde{D}_{v X} \rho Y, \rho Z\right)=v X \cdot g(\rho Y, \rho Z)+g(\rho Y, \rho[Z, v X])+g(\rho Z, \rho[v X, Y])-\left(\widetilde{D}_{v X} g\right)(\rho Y, \rho Z)$.

Hence, using Theorem 4(a) of [3], the above equation implies that

$$
2 g\left(\widetilde{D}_{v X} \rho Y, \rho Z\right)=2 g\left(\nabla_{v X} \rho Y, \rho Z\right)-\left(\widetilde{D}_{v X} g\right)(\rho Y, \rho Z) .
$$

Consequently,

$$
\begin{aligned}
2 g(\widetilde{\mathbf{T}}(v X, h Y), \rho Z) & =2 g\left(\widetilde{D}_{v X} \rho Y-\rho[v X, h Y], \rho Z\right) \\
& =2 g\left(\nabla_{v X} \rho Y-\rho[v X, h Y], \rho Z\right)-\left(\widetilde{D}_{v X} g\right)(\rho Y, \rho Z) \\
& =2 g(\mathbf{T}(v X, h Y), \rho Z)-\left(\widetilde{D}_{v X} g\right)(\rho Y, \rho Z) .
\end{aligned}
$$

From which, taking axiom (b) into account, we get:

$$
\left(\widetilde{D}_{v X} g\right)(\rho Y, \rho Z)=2 g(\mathbf{T}(v X, h Y), \rho Z) .
$$

Hence, (2) and (3), we obtain

$$
\widetilde{D}_{v X} \rho Y=\nabla_{v X} \rho Y-\mathbf{T}(v X, h Y) .
$$

Now, using axiom (c) and noting that $K \circ J=\gamma$ and $K \circ h=0$, we get:

$$
\begin{aligned}
-L T(\rho X, \rho Y)=\widehat{\widetilde{P}}(h X, J Y) & =\widetilde{P}(h X, J Y) \bar{\eta} \\
& =-\widetilde{D}_{h X} \widetilde{D}_{J Y} \bar{\eta}+\widetilde{D}_{J Y} \widetilde{D}_{h X} \bar{\eta}+\widetilde{D}_{[h X, J Y]} \bar{\eta} \\
& =-\widetilde{D}_{h X} \rho Y+K[h X, J Y] .
\end{aligned}
$$

From which,

$$
\widetilde{D}_{h X} \rho Y=K[h X, J Y]+L T(\rho X, \rho Y) .
$$

Using the definition of the $(v) h v$-torsion $\widehat{P}$, (5) may also be written in the form:

$$
\widetilde{D}_{h X} \rho Y=\nabla_{h X} \rho Y+\widehat{P}(\rho X, \rho Y)+L T(\rho X, \rho Y) .
$$

Consequently, from (4) and (6), the full expression of $\widetilde{D}_{X} \bar{Y}$ is given by:

$$
\widetilde{D}_{X} \bar{Y}=\nabla_{X} \bar{Y}+\widehat{P}(\rho X, \bar{Y})+L T(\rho X, \bar{Y})-T(K X, \bar{Y}) .
$$

Hence $\widetilde{D}_{X} \bar{Y}$ is uniquely determined by the right-hand side of (7).

To prove the existence, we define $\widetilde{D}$ by the requirement that (7) holds or, equivalently, (4) and (6) hold. Then, using the properties of Cartan connection $\nabla$ and the results of [3], it is not difficult to show that the connection $\widetilde{D}$ satisfies the conditions (a), (b), and (c).

In view of the above theorem, we have the following results:

Corollary 3 The $\widetilde{D}$-connection is explicitly expressed in terms of the Cartan connection $\nabla$ in the form:

$$
\widetilde{D}_{X} \bar{Y}=\nabla_{X} \bar{Y}+\widehat{P}(\rho X, \bar{Y})+L T(\rho X, \bar{Y})-T(K X, \bar{Y}) .
$$

In particular, we have:
(a) $\widetilde{D}_{\gamma \bar{X}} \bar{Y}=\nabla_{\gamma} \bar{Y}-T(\bar{X}, \bar{Y})=\rho[\gamma \bar{X}, \beta \bar{Y}]$.
(b) $\widetilde{D}_{\beta \bar{X}} \bar{Y}=\nabla_{\beta} \bar{X}+\widehat{Y}(\bar{X}, \bar{Y})+L T(\bar{X}, \bar{Y})=K[\beta \bar{X}, \gamma \bar{Y}]+L T(\bar{X}, \bar{Y})$.

Concerning the metricity properties of $\widetilde{D}$, we havec 
Corollary 4 The $\widetilde{D}$-connection has the properties:

(a) $\left(\widetilde{D}_{\gamma \bar{X}} g\right)(\bar{Y}, \bar{Z})=2 T(\bar{X}, \bar{Y}, \bar{Z})$.

(b) $\left(\widetilde{D}_{\beta \bar{X}} g\right)(\bar{Y}, \bar{Z})=-2 L T(\bar{X}, \bar{Y}, \bar{Z})-2 \widehat{P}(\bar{X}, \bar{Y}, \bar{Z})$,

where $\widehat{P}(\bar{X}, \bar{Y}, \bar{Z}):=g(\widehat{P}(\bar{X}, \bar{Y}), \bar{Z})$.

Remark 1 In view of the above proposition, one can show that a Finsler manifold $(M, L)$ is Riemannian if and only if $\widetilde{D}_{\gamma \bar{X}} g=0$. Moreover, $(M, L)$ is Landsbergian if and only if $\left(\widetilde{D}_{\beta \bar{X}} g\right)(\bar{Y}, \bar{Z})=-2 L T(\bar{X}, \bar{Y}, \bar{Z})$.

Proposition 3 The Curvature tensors of the $\widetilde{D}$-connection are given by:

(a) $\widetilde{S}(\bar{X}, \bar{Y}) \bar{Z}=S(\bar{X}, \bar{Y}) \bar{Z}-\mathfrak{U}_{\bar{X}, \bar{Y}}\{T(\bar{X}, T(\bar{Y}, \bar{Z}))\}$.

(b) $\widetilde{P}(\bar{X}, \bar{Y}) \bar{Z}=P(\bar{X}, \bar{Y}) \bar{Z}+L\left(\nabla_{\gamma \bar{Y}} T\right)(\bar{X}, \bar{Z})+\left(\nabla_{\gamma} \widehat{Y} \widehat{P}\right)(\bar{X}, \bar{Z})$

$$
\begin{aligned}
& +\ell(\bar{Y}) T(\bar{X}, \bar{Z})+L T(T(\bar{Y}, \bar{X}), \bar{Z})+\widehat{P}(T(\bar{Y}, \bar{X}), \bar{Z})-\nabla_{\beta} T(\bar{Y}, \bar{Z}) \\
& +T\left(\bar{Y}, \nabla_{\beta \bar{X}} \bar{Z}\right)-L T(\bar{Y}, T(\bar{X}, \bar{Z}))-T(\bar{Y}, \widehat{P}(\bar{X}, \bar{Z})) \\
& +L T(\bar{X}, T(\bar{Y}, \bar{Z}))+\widehat{P}(\bar{X}, T(\bar{Y}, \bar{Z}))-T(K[\gamma \bar{X}, \gamma \bar{Y}], \bar{Z}) .
\end{aligned}
$$

(c) $\widetilde{R}(\bar{X}, \bar{Y}) \bar{Z}=R(\bar{X}, \bar{Y}) \bar{Z}-T(\widehat{R}(\bar{X}, \bar{Y}), \bar{Z})-\mathfrak{U}_{\bar{X}, \bar{Y}}\left\{L\left(\nabla_{\beta} \bar{X}^{T}\right)(\bar{Y}, \bar{Z})\right.$

$$
\begin{aligned}
& +\left(\nabla_{\beta} \widehat{X}\right)(\bar{Y}, \bar{Z})+L^{2} T(\bar{X}, T(\bar{Y}, \bar{Z}))+L \widehat{P}(\bar{X}, T(\bar{Y}, \bar{Z})) \\
& +L T(\bar{X}, \widehat{P}(\bar{Y}, \bar{Z}))+\widehat{P}(\bar{X}, \widehat{P}(\bar{Y}, \bar{Z}))\},
\end{aligned}
$$

where $\ell(\bar{X}):=L^{-1} g(\bar{X}, \bar{\eta})$.

Remark 2 In view of the above Proposition, one can conclude that the v-curvature $\widetilde{S}$ vanishes and $\widehat{\widetilde{R}}(\bar{X}, \bar{Y})=\widehat{R}(\bar{X}, \bar{Y})$.

Using Proposition 3, we have the following properties of the hv-curvature and h-curvature of the $\widetilde{D}$-connection.

Proposition 4 The $h v$-curvature tensor $\widetilde{P}$ of $\widetilde{D}$-connection has the properties:
(a) $\widehat{\widetilde{P}}(\bar{X}, \bar{Y})=-L T(\bar{X}, \bar{Y})$,
(b) $\widetilde{P}(\bar{X}, \bar{Y}, \bar{Z}, \bar{W})+\widetilde{P}(\bar{X}, \bar{Y}, \bar{W}, \bar{Z})=2\left(\widetilde{D}_{\beta \bar{X}} T\right)(\bar{Y}, \bar{Z}, \bar{W})+2\left(\widetilde{D}_{\gamma} \widehat{\bar{P}}\right)(\bar{X}, \bar{Z}, \bar{W})$
(c) $\widetilde{P}(\bar{X}, \bar{Y}) \bar{Z}=\widetilde{P}(\bar{Z}, \bar{Y}) \bar{X}$, $2\left(\widetilde{D}_{\gamma \bar{Y}} L T\right)(\bar{X}, \bar{Z}, \bar{W})-2 T(\widehat{\widetilde{P}}(\bar{X}, \bar{Y}), \bar{Z}, \bar{W})$,
(d) $\left(\widetilde{D}_{\gamma \bar{X}} \widetilde{P}\right)(\bar{Y}, \bar{Z}, \bar{W})=\left(\widetilde{D}_{\gamma \bar{Z}} \widetilde{P}\right)(\bar{Y}, \bar{X}, \bar{W})$.

Proposition 5 The h-curvature tensor $\widetilde{R}$ of $\widetilde{D}$-connection satisfies the following properties:
(a) $\widetilde{R}(\bar{X}, \bar{Y}, \bar{Z}, \bar{W})=-\widetilde{R}(\bar{Y}, \bar{X}, \bar{Z}, \bar{W})$,
(b) $\widehat{\widetilde{R}}(\bar{X}, \bar{Y})=\widehat{R}(\bar{X}, \bar{Y})=-K \Re(\beta \bar{X}, \beta \bar{Y})$,
(c) $\widetilde{R}(\bar{X}, \bar{Y}, \bar{Z}, \bar{W})+\widetilde{R}(\bar{X}, \bar{Y}, \bar{W}, \bar{Z})=2 \mathfrak{A}_{\bar{X}, \bar{Y}}\left\{L\left(\widetilde{D}_{\beta \bar{Y}} T\right)(\bar{X}, \bar{Z}, \bar{W})+\left(\widetilde{D}_{\beta \bar{Y}} \widehat{P}\right)(\bar{X}, \bar{Z}, \bar{W})\right\}$
(d) $\mathfrak{S}_{\bar{X}, \bar{Y}, \bar{Z}}\{\widetilde{R}(\bar{X}, \bar{Y}) \bar{Z}\}=0$, $-2 T(\widehat{R}(\bar{X}, \bar{Y}), \bar{Z}, \bar{W})$,
(e) $\mathfrak{S}_{\bar{X}, \bar{Y}, \bar{Z}}\left\{\left(\widetilde{D}_{\beta \bar{X}} \widetilde{R}\right)(\bar{Y}, \bar{Z}, \bar{W})+\widetilde{P}(\bar{X}, \widehat{R}(\bar{Y}, \bar{Z})) \bar{W}\right\}=0$,
(f) $\quad\left(\widetilde{D}_{\gamma \bar{X}} \widetilde{R}\right)(\bar{Y}, \bar{Z}, \bar{W})=\left(\widetilde{D}_{\beta \bar{Z}} \widetilde{P}\right)(\bar{Y}, \bar{X}, \bar{W})-\left(\widetilde{D}_{\beta \bar{Y}} \widetilde{P}\right)(\bar{Z}, \bar{X}, \bar{W})$ $-L \widetilde{P}(\bar{Z}, T(\bar{Y}, \bar{X})) \bar{W}+L \widetilde{P}(\bar{Y}, T(\bar{Z}, \bar{X})) \bar{W}$,

where $\mathfrak{R}$ is the curvature of Barthel connection. 


\section{Acknowledgements}

The author express his sincere thanks to the reviewers and Professor Nabil L. Youssef for their valuable suggestions and comments

\section{Authors' contributions}

AS, mentioned in the first page, achieved this work. The author read and approved the final manuscript.

\section{Funding}

Not applicable.

\section{Availability of data and materials}

Not applicable.

\section{Competing interests}

Not applicable.

Received: 2 Mar 2019 Accepted: 9 July 2019

Published online: 04 September 2019

\section{References}

1. Bao, D., Chern, S. S., Shen, Z.: An introuduction to Riemann-Finsler geometry. Springer-Verlag, Berlin (2000)

2. Miron, R., Anastasiei, M.: The geometry of Lagrange spaces: theory and applications. Kluwer Acad. Publ. (1994)

3. Youssef, N. L., Abed, S. H., Soleiman, A.: Cartan and Berwald connections in the pullback formalism. Algebras Groups Geometries. 25, 363-386 (2008). arXiv: 0707.1320 [math. DG]

4. Youssef, N. L., Abed, S. H., Soleiman, A.: A global approach to the theory of connections in Finsler geometry. Tensor N.S. 71, 187-208 (2009). arXiv: 0801.3220 [math.DG]

5. Youssef, N. L., Abed, S. H., Soleiman, A.: Geometric objects associated with the fundumental connections in Finsler geometry. J. Egypt. Math. Soc. 18, 67-90 (2010). arXiv: 0805.2489 [math.DG]

6. Akbar-Zadeh, H.: Initiation to global Finsler geometry. Elsevier Science (2006)

7. Dazord, P.: Propriétés globales des géodésiques des espaces de Finsler, Thèse d'Etat. Publ. Dept. Math. (1969)

8. Soleiman, A.: Recurrent Finsler manifolds under projective change. Int. J. Geom. Meth. Mod. Phys. 13, 1650126 (2016). 10 pages

9. Tamim, A. A.: General theory of Finsler spaces with applications to Randers spaces, Ph. D. Thesis. Cairo University (1991)

10. Youssef, NL: Sur les tenseurs de courbure de la connexion de Berwald et ses distributions de nullité. Tensor, N.S. 36 275-280 (1982)

11. Grifone, J.: Structure présque-tangente et connexions, I. Ann. Inst. Fourier. 22, 287-334 (1972). Grenoble

12. Grifone, J.: Structure presque-tangente et connexions, II. Ann. Inst. Fourier. 22, 291-338 (1972). Grenoble

13. Klein, J., Voutier, A.: Formes extérieures génératrices de sprays. Ann. Inst. Fourier. 18, 241-260 (1968). Grenoble

14. Youssef, N. L., Abed, S. H., Soleiman, A.: A global approach to the theory of special Finsler manifolds. J. Math. Kyoto Univ. 48, 857-893 (2008). arXiv: 0704.0053 [math. DG]

\section{Publisher's Note}

Springer Nature remains neutral with regard to jurisdictional claims in published maps and institutional affiliations.

\section{Submit your manuscript to a SpringerOpen ${ }^{\circ}$ journal and benefit from:}

- Convenient online submission

Rigorous peer review

- Open access: articles freely available online

High visibility within the field

- Retaining the copyright to your article

Submit your next manuscript at $\gg$ springeropen.com 